\title{
A study of the factors affecting the outcome of vertebromedullary injuries
}

\author{
M Hanci ${ }^{1}$, T Kuday ${ }^{2}$, AÇ Sarioğlu ${ }^{3}$ and M Uzan ${ }^{4}$ \\ ${ }^{1}$ Consultant Neurosurgeon, ${ }^{2}$ Associate Professor of Physical Therapy and Rehabilitation, ${ }^{3}$ Professor of \\ Neurosurgery, ${ }^{4}$ Resident of Neurosurgery, Istanbul University Cerrahpaşa Medical Faculty, Department of \\ Neurosurgery, Aksaray, Istanbul, Turkey
}

\begin{abstract}
Between 1982 and 1991, 194 patients were treated for cervical vertebromedullary injuries in the Department of Neurosurgery, Cerrahpasa Medical Faculty of the University of Istanbul. The data were evaluated with the multiple regression method using the SPSS/PC statistical package to predict the factors influencing the outcome. In the initial neurological examination, motor deficit was detected in $63.4 \%$ of the patients, sensorial deficit was seen in $53.6 \%$ of the patients and respiratory failure in $12.9 \%$. Of these patients $46.4 \%$ were treated surgically and $53.6 \%$ medically. Evaluating the findings and outcomes with the Frankel scale, no change in neurological status was detected in $44.8 \%$ of the patients. Improvement was detected in $36.1 \%$ and deterioration in $19.1 \%$, the mortality rate being $18.6 \%$. It is concluded that the main factor predicting the outcome of patients with vertebromedullary injury is the initial neurological status $(P<0.0001)$.
\end{abstract}

Keywords: spinal injury; prediction, cervical spine

\section{Introduction}

Severe injury to the spinal cord often results in death or irreversible motor and sensory deficits. There may not only be potentially unstable fractures but also difficult clinical conditions which are complicated by widely variable physiological parameters which require explicit explanations for the prognosis. Some colleagues have argued that certain factors affecting outcome may be surgical treatment versus conservative treatment, age, and type of the injury. Regarding these hypotheses, we examined our patients' outcomes by statistical analysis and tried to accept or reject some of these arguments.

\section{Material and methods}

Between 1982 and 1991, 194 patients with cervical spinal cord injury were treated at the University of Istanbul Cerrahpasa Medical Faculty, Department of Neurosurgery. Most of the patients were aged 20-30 and $30-40$ years $(25.3 \%$ and $21.6 \%)$. There were a few pediatric patients and the incidence declined with advanced age. There were $70.6 \%(n=137)$ male and $29.4 \%(n=57)$ female. Regarding aetiology, motor vehicle accidents were most prominent $(41.2 \%)$ followed by falls from heights $(34 \%)$ and diving accidents $(18 \%)$. Penetrating injuries were responsible in only $3.1 \%$ of the cases. The victims of falls from heights were mostly elderly male patients, whereas injuries resulting from motor vehicle accidents occurred mostly

Correspondence: Murat Hanci, PO Box 792, 8220 Şişli, Istanbul, Turkey among young adults with no gender dominance. Penetrating injuries were five times more frequent in male patients.

The period between the time of accident and admittance to our hospital was less than $24 \mathrm{~h}$ in $52.6 \%$ of the patients. Thirty seven patients were transferred from other local hospitals or had a delay in diagnosis, and were admitted 1 week to 1 month after the accident.

There was no motor deficit in $36.6 \%$ of the patients, where deficits differing from monoparesis to tetraplegia were detected in $63.4 \%$ of the patients. No sensory deficits were detected in $46.4 \%$ of the patients. For the patients with sensory deficits, $6.2 \%$ had deficit localised to only one extremity, in $4.6 \%$ of the patients to one half of the body, in $14 \%$ of the patients it extended to the thoracic segments and in $27.8 \%$ it extended to cervical segments. Respiratory failure was detected by clinical and laboratory studies and was present in $12.9 \%$ of the patients.

$\mathrm{X}$-ray studies were unremarkable except for cervical spondylosis in eight patients. Forty five had upper cervical and 148 had lower cervical lesions. The most common site of injury was C2 (28 cases) in those with upper cervical spine lesions and C5 (58 cases) in those with lower cervical spine lesions.

Conservative treatment was carried out in $53.6 \%$ of the patients and the remainder were treated surgically. In those with incomplete lesions both methods were equally used. In patients with no neurological deficits conservative treatment was given; however in patients showing spinal instability, operative procedures were chosen. Most of the patients with a complete medullary 
injury presented lesions which narrowed the spinal canal and produced bilateral locked facets and displaced compression fractures. In the patients in which no clinical relief was obtained by traction, surgical procedures were carried out for decompression and/or reduction and stabilisation.

\section{Results}

At the initial examination, $35.1 \%$ of the patients showed no neurological deficits, (E grade according to Frankel scale), in $19.1 \%$ of the patients complete lesions, and in $45.8 \%$ of the patients incomplete lesions (B, C, D) were found. At the end of the follow-up period extending from 8 months to 11 years, $54.6 \%$ of the cases were found to be at Frankel E grade. In 37 patients complete lesion was thought to be found; 26 of them died, six had no change in their neurological status, two had 1 grade, two had 2 grades and one had 4 grades of improvement. Eighty nine patients had incomplete lesions, 14 of whom had no improvement in their neurological status. Ten patients died. Thirty eight patients had 1 grade, 22 had 2 grades and five had 3 grades of improvement. The neurological status deteriorated in 37 patients (Figure 1). All of these patients except one, who had a postoperative monoparesis, died from deterioration of the medullary injury or from various complications. Of those with complete lesions, three patients died from asystole unresponsive to resuscitation, two from massive gastrointestinal haemorrhage, 11 patients from gram negative pneumonia during mechanical ventilation, one from sepsis from a decubitus ulcer and nine patients from vasomotor collapse secondary to progression of the medullary injury. Of those with incomplete lesions, one died from postoperative adult respiratory distress syndrome (ARDS), one patient from airway obstruction, one from autonomic hyperreflexia during a myelographic study, two from sepsis due to urinary system infection, one pregnant patient from eclampsia, three patients from accompanying traumatic lesions and one from iatrogenic medullary damage during intervention. The incidence of neurological deterioration was higher than would have been expected. We think that this was due

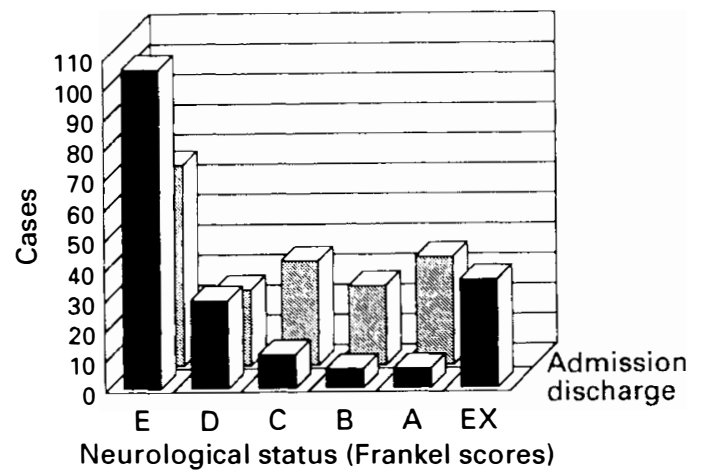

Figure 1 Graph showing distribution of Frankel scores of 194 patients at admission and discharge. to the complications mentioned above. The overall mortality was $18.6 \%$. The ratio of the grade $\mathrm{E}$ patients which was $35.1 \%$ on admission, finally rose to $54.6 \%$.

The data which are documented in detail above were analysed statistically to determine the factors influencing the outcome. The patient data were evaluated by the multiple regression method using the SPSS/PC statistical package. The neurological status at admission according to Frankel scale is the main factor determining the outcome $(P<0.0001)$.

\section{Discussion}

The prognosis of spinal injuries correlates with the severity of the medullary lesion. As Bedbrook ${ }^{1}$ stated, the neurological status occurs at the time of the accident, in other words, prognosis is determined from the beginning. The authors believe that there is no chance of recovery for patients with complete lesions. Patients who did show neurological recovery had incomplete lesions which were misdiagnosed at the very beginning. For these reasons, one must not decide whether a lesion is complete or incomplete before 3 weeks in the case of patients with paraplegia and 5 weeks in those with tetraplegia. ${ }^{2}$ In his experimental studies, Guha ${ }^{3}$ showed that surgical decompression procedures made within the first $4 \mathrm{~h}$ following the injury may have some improvement, but even so, the severity of the trauma is the main determinant for the prognosis. Ersmark ${ }^{4}$ stated that there is no correlation between the method of treatment and the outcome. According to Wilmont ${ }^{5}$ there is no relationship between the method of treatment and the period needed for rehabilitation. He also noted some improvement in $18 \%$ of patients at the end of rehabilitation, but according to him this must not be attributed to surgical treatment. As Bedbrook ${ }^{6}$ stated, improvement in the neurological status is the result of long term sensorial stimulation and isometric muscle exercises. Yarkony ${ }^{7}$ and Geisler ${ }^{8}$ pointed out that in patients with incomplete lesions, movement ability, capacity of meeting the daily requirements, survival rate and quality of life depends on the rehabilitation programme applied at home. In view of this many centres have abandoned laminectomy surgery, which has no influence on the neurological status and is limited as a surgical procedure to obtain stability. ${ }^{6}$ An important question for those with spinal injuries is to what extent one must go to determine the diagnosis? We support Scher's ${ }^{9}$ opinion that detailed radiological examinations which help to determine the type of injury and may identify small fractured fragments, may be harmful for the patient, and that there is no relationship between the radiological findings and the medullary injury.

\section{Conclusion}

Statistical analysis revealed that the patient's condition on admission is the most important factor ( $P<0.00001)$ influencing the outcome. Other factors such as the level of bone lesion $(P=0.0882-0.8921)$, 
method of treatment $(P=0.0865)$ and time interval $(P=0.0263)$, have no statistical significance and play only secondary roles in prognosis. We propose to carry out surgical interventions in patients with primary or secondary spinal instability, and to begin a rehabilitation programme as soon as possible for our patients.

\section{Acknowledgements}

We would like to thank Deniz Gökçe (PhD), Emre Denizci (Eng) and Cem Alhan MD (Consultant in Cardiovascular Surgery) for their valuable assistance in preparing this paper.

\section{References}

1 Bedbrook GM, Sakae T. A review of cervical spine injuries with neurological disfunction. Paraplegia 1982; 20: 321-333.
2 Michaelis LS. Prognosis of spinal cord injury. Handbook of Clinical Neurology Vol. 26. In: Vinken PJ, Bruyn GW (eds). Amsterdam: North Holland Publishing Company Amsterdam, 1976: $307-312$.

3 Guha A, Tator $\mathrm{CH}$, Endrenyi L, Piper I. Depression of the spinal cord improves recovery after acute experimental spinal cord compression injury. Paraplegia 1987; 25: 324-339.

4 Ersmark H, Dalen N, Kalen R. Cervical spine injuries: A follow-up of 332 patients. Paraplegia 1990; 28: 25-40.

5 Wilmont CB, Hall KM. Evaluation of the acute management of tetraplegia: Conservative versus surgical treatment. Paraplegia 1986; 24: 148-153.

6 Bedbrook GM. Spinal injuries with tetraplegia and paraplegia. J Bone Joint Surg Br 1979; 61: 267-284.

7 Yarkony GM, Roth EJ, Heinemann AW. Benefits of rehabilitation for traumatic spinal cord injury. Arch Neurol 1987; 44: 93-96.

8 Geisler WO, Jousse AT, Jones MW, Breithaupt D. Survival in traumatic spinal cord injury. Paraplegia 1983; 21: 364-373.

9 Scher AT. Is the pattern of neurologic damage of diagnostic value in the radiological assessment of acute cervical spine injury. Paraplegia 1981; 19: 248-252. 